195 CRISPR/CAS9 GENE EDITING REVEALS NOVEL TERTIARY CONSTRAINTS IN CLUSTERED MIRNA PROCESSING

${ }^{1}$ Lazaros Lataniotis* ${ }^{2}$ Andreas Albrecht, ${ }^{3}$ Fatma Kok, ${ }^{4}$ Clinton Monfries, ${ }^{5}$ Lorena Benedetti, ${ }^{3}$ Nathan D Lawson, ${ }^{4}$ Simon M Hughes, ${ }^{6}$ Kathleen Steinhofel, ${ }^{1}$ Manuel Mayr, ${ }^{1}$ Anna Zampetaki. 'King's British Heart Foundation Centre, King's College London; ${ }^{2}$ Middlesex University; ${ }^{3}$ University of Massachusetts Medical School; ${ }^{4}$ King's College London, Randall Division; ${ }^{5}$ King's College London, Division of Cancer Studies; ${ }^{6}$ King's College London, Informatics Department

\subsection{6/heartjnl-2017-311726.193}

Introduction MicroRNAs (miRNAs) play an important role in the cellular function. They often form families, with members sharing high sequence homology, a property that hampers miRNA research as there is a lack of elegant tools for specific miRNA manipulation.

Objective To establish a reliable workflow for miRNA inhibition using genome editing.

Methods and Results This study focused on miR-195, a member of the miR-15 family and employed the CRISPR-Cas9 system. To this end we generated mouse vascular smooth muscle cells (VSMCs) stably expressing Cas9 nuclease. Cells were then transfected with an in vitro transcribed single guide RNA targeting the miR-195 stem loop. T7 endonuclease I assay (T7EI) and Sanger sequencing confirmed efficient editing. QPCR demonstrated effective decrease of miR-195 but not of miR-15a and miR-16, two highly expressed members of the miR-15 family in VSMCs. Surprisingly the expression of miR497 was also decreased in edited cells. Noteworthy, miR-195 and miR-497 form a miRNA cluster and are co-transcribed as a primary miRNA. No gene editing was detected by T7EI and sequencing of the mir-497 genomic locus. Computational simulation predicted that mutations of the miR-195 stem loop led to changes in the three dimensional structure of the primary miR-497 195 transcript that could affect its processing to mature miRNAs.

Similar findings were obtained the miR-143 145 cluster that encodes miR-143 and miR-145a, two miRNAs that do not belong to the same family, show no sequence homology and play a pivotal role in vascular biology. Specific targeting of the mir-145a locus effectively inhibited the expression of both miR-143 and miR-145a while no genomic editing was observed for the mir-143 locus. Noteworthy, the expression of Carmn, a long non coding RNA in the vicinity of the miR143 145 cluster that constitutes an independent transcription unit did not differ in miR-145a edited cells confirming that only the primary miRNA transcript is affected. On the contrary, gene editing in the miR-17 92 and miR-106b 25 clusters, two miRNA clusters with a key function in the cardiovascular system, resulted in targeted miRNA inhibition. MiR-18a and miR-25 were targeted on each cluster, respectively. Specific editing only for the intended miRNA locus was observed and QPCR quantification indicated inhibition of the edited miRNA. No effect on the expression of other miRNAs occurred, both for cluster miR-17 92 and miR-106b 25.

Detailed analysis of the gene editing in the four clusters revealed that the unintended inhibition of miRNA expression in the cluster coincides with disruption of sequence motifs of the terminal loop of the targeted hairpin, suggesting that these elements are critical for the maturation not only of individual hairpins but the entire primary transcript in miRNA clusters. Conclusions CRISPR/Cas9 emerged as a powerful tool that can offer novel insights into the role of miRNAs in cardiovascular diseases.

\section{FUNCTIONAL HIGH-THROUGHPUT SCREENING IDENTIFIES MICRORNA-26B AS PRO-SURVIVAL AND ANGIOGENEIC FACTOR FOR ENDOTHELIAL CELLS}

${ }^{1}$ David Mellis*, 'Lorraine Rose, ${ }^{1}$ Andrea Martello, ${ }^{2}$ Ayman Al-Haj Zen, ${ }^{1}$ Andrea Caporali. ${ }^{1}$ University/BHF Centre for Cardiovascular Science, QMRI, University of Edinburgh; ${ }^{2}$ Wellcome Trust Centre for Human Genetics, Division of Cardiovascular Medicine, Radcliffe Department of Medicine, University of Oxford

\subsection{6/heartjnl-2017-311726.194}

Critical limb ischaemia (CLI) is a serious form of peripheral artery disease where patients suffer from reduced arterial blood flow and subsequent loss of tissue viability. Therapeutic angiogenesis uses pro-angiogenic factors to stimulate new blood vessels formation to bypass existing blockages. Despite promising pre-clinical data, the outcome of clinical trials using single proangiogenic growth factors remains inconclusive. MicroRNAs (miRNAs) are small non-coding RNAs that orchestrate genetic networks by modulating simultaneous gene expression, thus suitable for therapeutic regulation of postischaemic angiogenesis. The aim of this study is to identify novel miRNAs that can influence vascular endothelial cells (EC) function and identify their relevant target genes. High content screening (HCS) of human miRNA mimic library is an emerging approach that utilises robotic microscopy platforms along with unbiased image analysis algorithms to generate quantitative data. Therefore, we used HCS to identify miRNAs that regulate EC proliferation and establish their potential as a therapeutic target for CLI. High throughput screening of over 1500 unique microRNA mimics revealed miR-26b as a top candidate for regulating EC functions. The overexpression of miR-26b significantly increased the proliferation and migration of ECs. Furthermore, it increased cell survival and importantly enhanced EC tube formation and branching morphogenesis. Stimulating ECs with the growth factor VEGFA increased EC proliferation associated with increased miR-26b expression. Expression analysis revealed an increase in endothelial cell tip enrichment genes including FLT4, ESM1, Ang-2 and PDGFB in EC overexpressing miR26b. In-vivo, Q-PCR analysis confirmed an increase level of miR-26b expression in EC sorted form limb muscles 3 days post-ischaemia. Furthermore, we tested the effects on in vivo angiogenesis using the Matrigel implant model. Histological analysis at day 10 with the implants confirmed miR-26b mimics increased the vessel density. After confirming the important pro-survival and pro-angiogenic role miR-26b has in ECs, we used HCS to identify downstream target genes. We found that Phosphatase and Tensin homolog (PTEN) and phosphatase gene PPP2R2A are direct targets of miR-26b and their regulation determined the phenotype of miR-26b in ECs. MicroRNA-26b was for the first time identified as a pro-survival and pro-angiogenic factor for EC by the use of novel HCS techniques. We believe miR-26b is an excellent therapeutic target for the treatment of CLI and we continue to investigate and characterise its role in post-ischaemic angiogenesis.

197 THE CARDIAC STRESS RESPONSE PROTEIN MS1- A NEW TRANSCRIPTION FACTOR REGULATING HYPERTROPHY?

Mark Pfuhl* . King's College London

10.1136/heartjnl-2017-311726.195 
Introduction Ms1 (also known as STARS and ABRA) has been shown to act as an early stress response gene in processes as different as hypertrophy in skeletal and cardiac muscle and growth of collateral blood vessels. It is important for cardiac development in zebrafish and is upregulated in mouse models for cardiac hypertrophy as well as in human failing hearts. Ms1 possesses actin binding sites at its C-terminus and is usually found in the cell bound to actin filaments in the cytosol or in sarcomeres. It is assumed that it activates SRF dependent gene expression in an indirect manner by shifting the equilibrium between cytosolic actin from $G$ to $F$. This releases MRTFs which can then translocate to the nucleus to bind to SRF.

Methods We used NMR spectroscopy to determine the structure of the only folded domain of Ms1 and characterised its binding to F-actin and DNA using a combination of biochemical and biophysical assays. To identify a specific DNA sequence we used the SELEX approach in vitro in combination with EMSA assays. This was followed up by monitoring Ms1 subcellular localisation in neonatal rat cardiomyocytes using detection of endogenous protein with a new antibody or overexpression of wild type and mutated proteins.

Results We determined the NMR structure of the only folded domain of Ms1 comprising the second actin binding site called actin binding domain 2 (ABD2, residues 294-375), and found that it is similar to the winged helix-turn-helix fold adopted mainly by DNA binding domains of transcriptional factors. In vitro experiments show specific binding of this domain, in combination with a newly discovered AT-hook motif located N-terminally, to the sequence (A/C/G)AAA(C/A). NMR and fluorescence titration experiments confirm that this motif is indeed bound specifically by the recognition helix. In neonatal rat cardiomyocytes endogenous Ms1 is found in the nucleus in a spotted pattern, reminiscent of PML bodies. In adult rat cardiomyocytes Ms1 is exclusively found in the sarcomere. A nuclear localisation site (NLS) in the N-terminus of the protein is required for nuclear localisation as shown by the abolition of nuclear translocation of over expressed protein in which the NLS was mutated. A phosphorylation site immediately N-terminal of this NLS could provide regulatory control of nuclear transport.

Conclusions Our data suggest that Ms1 has the potential to act directly in the nucleus through specific interaction with DNA in development and potentially as a response to stress in adult tissues. Ms1 could act in parallel to the established MRTF/SRF pathway to regulate the expression of hypertrophic genes directly which would explain recent findings from studies in C1C12 cells where inhibition of SRF signalling did not lead to a change in myotube differentiation rate

\section{HEART FAILURE INCREASES MITOCHONDRIAL S- NITROSYLATION}

${ }^{1}$ Emma Radcliffe*, ${ }^{2}$ Junhui Sun, ${ }^{2}$ Angel Aponte, ${ }^{1}$ David Eisner, ${ }^{2}$ Elizabeth Murphy, ${ }^{1}$ Andrew Trafford. 'University of Manchester; ${ }^{2}$ National Heart, Lung and Blood Institute, NIH

\subsection{6/heartinl-2017-311726.196}

Heart failure affects over 550000 people living in the United Kingdom (1) and roughly half of those present with reduced cardiac ejection fraction (2). The electron transport chain (ETC) within the mitochondria is the principle source of ATP within cardiac muscle. Consequently, the regulation of the ETC in heart failure represents a potential rate limiting step to cardiac contraction in the disease state. The post-translational modification of proteins by nitric oxide, S-nitrosylation (S-NO) has previously been shown to have inhibitor effects on complex I activity (3). The aim of this study is therefore to investigate the role of $\mathrm{S}-\mathrm{NO}$ and how this may regulate ETC activity in an ovine tachypaced model of heart failure.

Tachypacing induced heart failure resulted in an increase in left ventricular diameter $(3.10 \pm 0.06 \mathrm{~cm}$ to $4.04 \pm 0.13 \mathrm{~cm}$, $\mathrm{p}<0.01, \mathrm{n}=5$ ), a thinning of the left ventricular free wall (81 $\pm 10 \mathrm{~mm}$ to $26 \pm 3 \mathrm{~mm}, \mathrm{p}=0.01, \mathrm{n}=5$ ) and a reduction in left ventricular contractility $(0.47 \pm 0.01$ to $0.20 \pm 0.03, \mathrm{p}<0.01, \mathrm{n}=5)$. Mass spectrometry in combination with either tandem mass tags or S-NO resin assisted capture, was used to assess protein expression or S-NO abundance respectively. The total number of S-NO proteins identified increased from $232 \pm 18$ in control samples to $314 \pm 28.3$ in heart failure $(p=0.02, n=6)$. Of those identified in both groups $79 \%$ showed an increase in S-NO abundance in heart failure. Mitochondrial content per unit of protein was unaltered in heart failure $(\mathrm{p}=0.93, \mathrm{n}=5)$. However, $\mathrm{S}$-NO of several ETC complex subunits was increased in the disease state (eg. NDUFS1 $\uparrow 2.9$ fold, SDHA $\uparrow 1.2$ fold, UQCRH $\uparrow$ 1.1 fold, $n=6)$ in the absence of corresponding protein expression changes (eg. NDUFS1 $\downarrow 0.2$ fold, SDHA $\downarrow 0.07$ fold, $\mathrm{UQCRH}+0.2$ fold, $\mathrm{n}=5$ ). In vitro $\mathrm{S}-\mathrm{NO}$ of control mitochondria, using $0.2 \mathrm{mM} \mathrm{S}$-nitrosoglutathione, oxidative phosphorylation by $52 \%(\mathrm{p}<0.01, \mathrm{n}=5)$.

This study demonstrates that in heart failure there is a gross increase in the level of myocardial S-NO. Within the mitochondria, S-NO of electron transport chain proteins is also increased, having an inhibitory effect on ATP production. This work therefore provides a novel insight into how S-NO may contribute to the deterioration of cardiac contractile function in heart failure.

\section{REFERENCES}

1. Townsend NWJ, Bhatnagar P, Wickramasinghe K, Rayner M. Cardiovascular disease statistics 2014. London: British Heart Foundation; 2014.

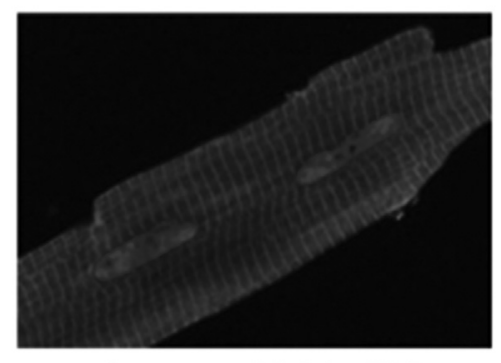

endogenous Ms1 in ARCs

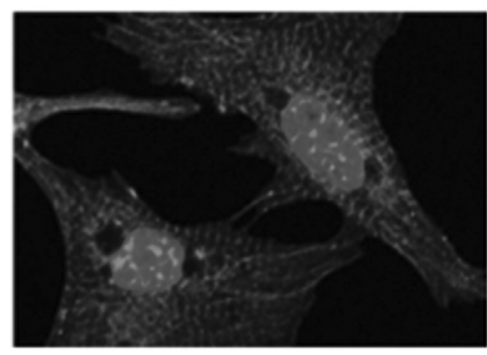

endogenous Ms1 in NRCs 